Pore Connectivity, Episodic Flow, and Unsaturated Diffusion in Fractured Tuff FY06 Annual Report

Qinhong Hu, R.P. Ewing, L. Tomutsa

November 14, 2006 
This document was prepared as an account of work sponsored by an agency of the United States Government. Neither the United States Government nor the University of California nor any of their employees, makes any warranty, express or implied, or assumes any legal liability or responsibility for the accuracy, completeness, or usefulness of any information, apparatus, product, or process disclosed, or represents that its use would not infringe privately owned rights. Reference herein to any specific commercial product, process, or service by trade name, trademark, manufacturer, or otherwise, does not necessarily constitute or imply its endorsement, recommendation, or favoring by the United States Government or the University of California. The views and opinions of authors expressed herein do not necessarily state or reflect those of the United States Government or the University of California, and shall not be used for advertising or product endorsement purposes.

This work was performed under the auspices of the U.S. Department of Energy by University of California, Lawrence Livermore National Laboratory under Contract W-7405-Eng-48. 


\title{
Pore Connectivity, Episodic Flow, and Unsaturated Diffusion in Fractured Tuff
}

\author{
"Max" Q. Hu ${ }^{1}$, R. P. Ewing ${ }^{2}$, and L. Tomutsa ${ }^{3}$ \\ ${ }^{1}$ Lawrence Livermore National Laboratory \\ ${ }^{2}$ Iowa State University \\ ${ }^{3}$ Lawrence Berkeley National Laboratory
}

In low-permeability unsaturated fractured rock, water flows predominantly through the interconnected fracture network, with some water imbibing into the neighboring matrix rock. Imbibition (driven by capillary pressure gradient) advectively transports radionuclides from fractures into the matrix. Diffusion (driven by concentration gradient) can diffusively transport radionuclides into the matrix (matrix diffusion), or from the matrix back into flowing fractures (back diffusion). Once in the matrix, sorbing radionuclides can sorb onto matrix rock. Such fracture-matrix interactions (with the interacting imbibition, diffusion, and sorption processes) tend to retard breakthrough of a radionuclide pulse released to the fracture network. A number of factors, such as initial matrix saturation, matrix pore connectivity, and intermittent flow events, will influence these interacting processes; these factors are investigated in this work.

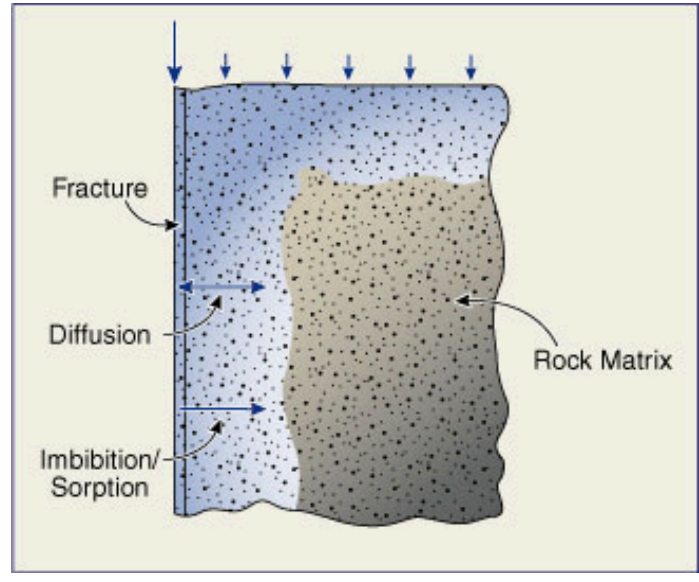

Figure 1. Schematic of fracture-matrix interactions in an unsaturated fractured rock.

\section{Accomplishments in FY 2006}

Preparation of initially moist samples: Partially-saturated cores were obtained, by equilibrating initially fully-saturated cores within relative humidity $(\mathrm{RH})$ chambers controlled by different over-saturated brines (Table 1) until they reached constant weights. Two RH chambers, with the RH values of $44 \%$ (mimic the low RH during the heat dissipation phase after nuclear waste package emplacement) and $98 \%$ (in situ condition of the vadose zone at Yucca Mountain), were used in this project. Matric potential, $P_{c}$, under each RH is calculated by the Kelvin equation (Table 1). The cores were placed inside the chamber, without direct contact with the solution. The relative humidity inside the chamber was verified using a calibrated hygrometer. The cores within the RH chambers were periodically weighed to monitor core weight loss until constant weight (and uniform saturation) was achieved, after which the initial water saturation was obtained based on the measured porosity. The equilibrated core samples were then used in the tests for tracer imbibition and unsaturated diffusion discussed later.

Table 1. Core saturation within two relative humidity chambers

\begin{tabular}{|c|c|c|}
\hline Chemicals & $\mathrm{K}_{2} \mathrm{CO}_{3}$ & $\mathrm{CaSO}_{4}$ \\
\hline $\mathrm{RH}(\%)$ & 44 & 98 \\
\hline $\mathrm{P}_{\mathrm{c}}(\mathrm{MPa})$ & -112.8 & -2.777 \\
\hline
\end{tabular}


Water imbibition and tracer transport: In order to probe for the pore connectivity in 10 UZ model layers (tsw34, tsw35, tsw36, tsw37, tsw38, tsw39, vitric subzone ch1, zeolitized ch1, vitric ch2-5, and zeolitized ch2-5) below the potential repository at Yucca Mountain, replicate measurements of water imbibition into initially dry tuff samples were conducted. For Topopah Spring welded tuff (tsw34), vitric Calico Hills (CHv), and zeolitized Calico Hills $(\mathrm{CHz})$ samples that are representative tuff below the potential repository, cores with several sample shapes (height vs. diameter) were employed to investigate the pore connectivity in these major tuff types with different mineralogy and chemical reactivity. Three different imbibition slope behaviors were seen as a result of different pore connectivities. In devitrified and zeolitized samples, slopes are sensitive to the sample's shape, with tall thin samples being more likely to have a slope of $1 / 4$ (anomalous diffusion behavior), sometimes followed by crossover to a slope of $1 / 2$ (classical). Short squat samples never display $1 / 4$-type behavior. These results confirm that the devitrified rock pore space is sparsely connected. Conversely, vitric Calico Hills tuff has well connected pore spaces, with the imbibition slopes showing only the $1 / 2$-type behavior even for the thin sample.

Tracer transport during imbibition into different antecedent water saturations (initially oven-dry, equilibrated at $44 \%$ and $98 \% \mathrm{RH}$ ) of $\mathrm{TSw}, \mathrm{CHv}$, and $\mathrm{CHz}$ have been performed. The tracer solution contains a suite of non-sorbing and sorbing tracers (including $\mathrm{ReO}_{4}^{-}$, radionuclides ${ }^{237} \mathrm{~Np}$ and ${ }^{242} \mathrm{Pu}$ ). We then used laser ablation interfaced with inductively coupled plasmamass spectrometry (LA / ICP-MS) to measure the fine-scale distribution of tracers with distance from the imbibing rock face.
Figure 2 shows the non-retarded transport of $\mathrm{ReO}_{4}^{-}$, and delayed transport from sorption for ${ }^{237} \mathrm{~Np}$ and ${ }^{242} \mathrm{Pu}$ tracers in TSw34 samples at different antecedent water saturations. For the imbibition experiment in initially dry core, the tracer $\mathrm{ReO}_{4}{ }^{-}$front is steep because a strong capillary force drives advective flow and tracer transport (Figure 2a). During imbibition into a moist medium, solutes will disperse by mixing with "old" water, and so a less sharp profile of $\mathrm{ReO}_{4}$ ' is observed (Figure 2c). In other words, dispersion becomes more pronounced for tracer transport under high initial saturation.
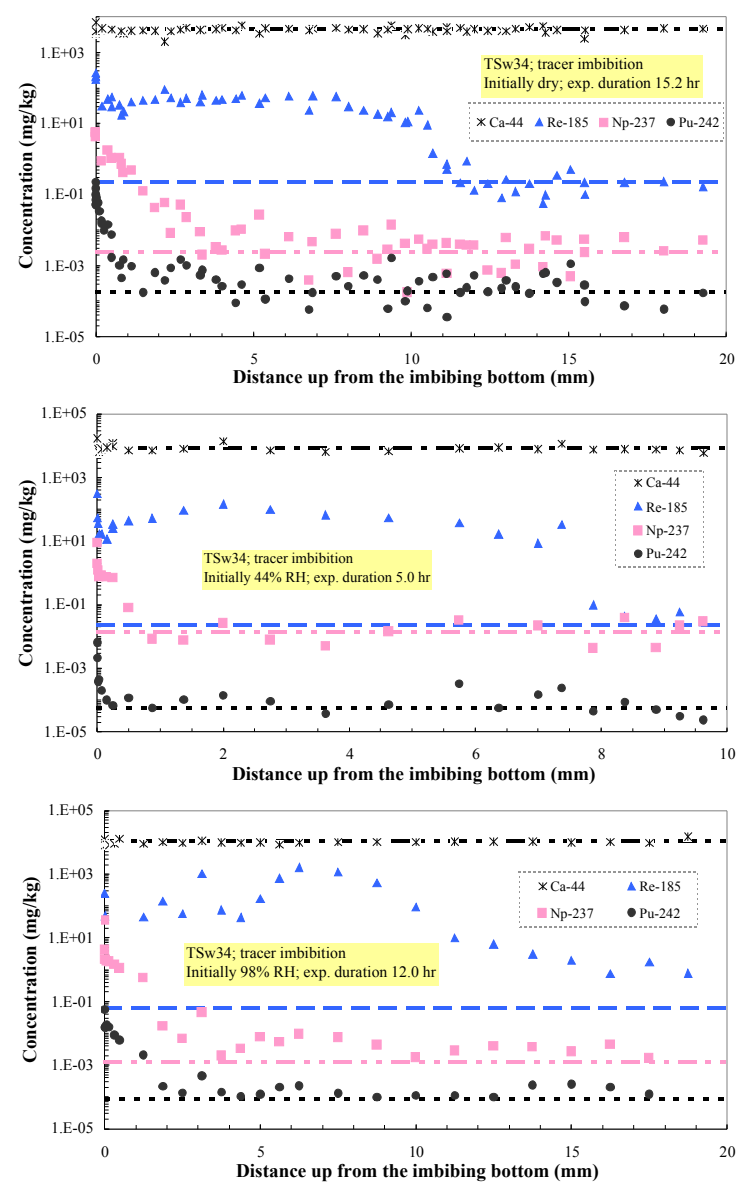

Figure 2. Tracer distribution after imbibing into initially (A) dry, (B) moist at $44 \% \mathrm{RH}$, and (C) moist at 98\% RH TSw34 cores. Also presented is the intrinsic rock element ${ }^{44} \mathrm{Ca}$ to show the uniform distribution. Dashed lines are the background levels for each tracer. 
The matrix potential as a function of moisture saturation, according to van Genuchten relationship, for three representative Yucca Mountain tuffs is shown in Figure 3.

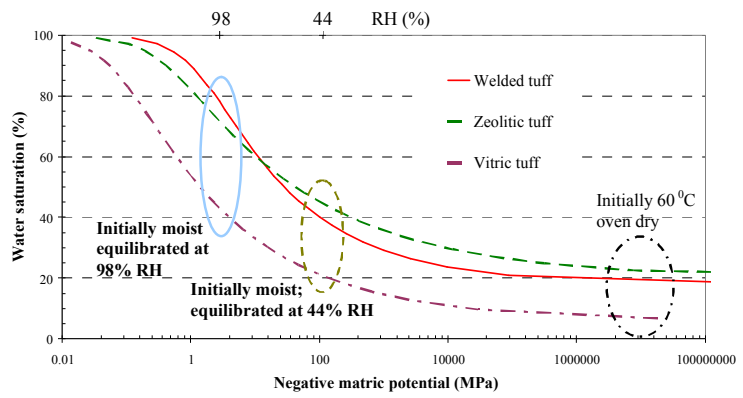

Figure 3. Moisture retention curves for representative vadose zone Yucca Mountain rock (Flint, 2003).

Synchrotron microtomography: Using the ALS microtomography beamline 8.3.2, we imaged a Berea sandstone with wellconnected pore spaces (Figure 4). We had planned to image and process the tuff samples to examine their pore connectivity, as well as the distribution of diffusing iodide into the tuff. However, various issues (broken camera, facility shut-down for beam upgrade) over the majority of past year have prevented our access to the ALS microtomography apparatus. The apparatus is expected to be up and running after January of 2007.

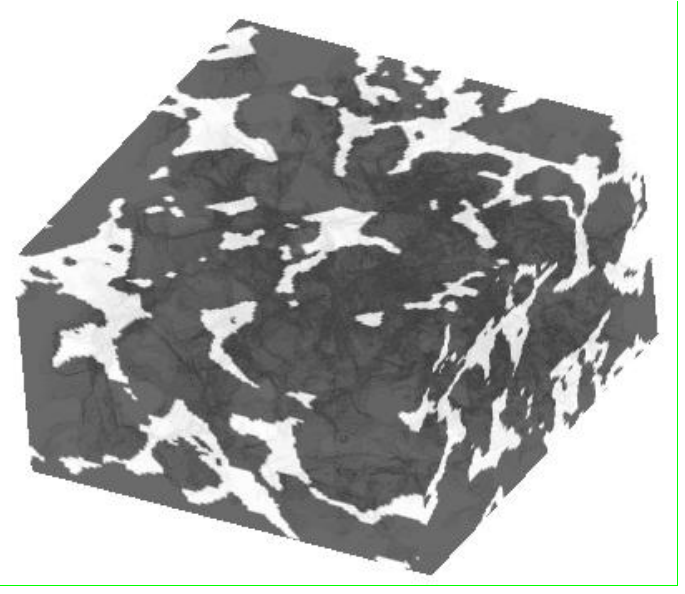

Figure 4. 3-D (1.1 $\mathrm{mm}$ each side) image of Berea sandstone; the pore space is white and the grains are black.

Gas diffusion: We have constructed an experimental set-up to conduct diffusive transport of two diffusing gases (helium and sulphur hexafluoride, $\mathrm{SF}_{6}$ ) with different diffusion coefficients. Gas concentration of these diffusing tracers (as well as nitrogen and argon in the air) was continuously monitored using the Membrane Inlet Mass Spectrometry (MIMS), which has a large dynamic measurement range, rapid data acquisition, and continuous real-time data display. Testing was performed for the reference porous rock, Berea sandstone. Analyses of the obtained breakthrough curves indicated the gas transport was not wholly diffusive; gas advection occurred because of its sensitive nature to slight pressure difference between up- and downstreams of the rock sample. Due to the limited resources, we discontinued the effort of modifying the setup to remove advection component. Instead, we focused our effort in the radionuclide diffusion tests in unsaturated rock.

Saturated and unsaturated tracer diffusion: Saturated diffusion tests were conducted to investigate tracer diffusion into fully watersaturated, with the help of vacuum pulling, cylindrical rock (TSw34, $\mathrm{CHv}$, and $\mathrm{CHz}$ ) samples. The samples, sitting on a Teflon mesh, were placed inside a chamber such that only the bottom portion (to minimize hydraulic head difference) was in contact with the tracer solution that was constantly stirring with a magnetic stirrer. The solution reservoir is as large as $1000 \mathrm{~mL}$, compared to $<1 \mathrm{~mL}$ pore volume of the samples, to maintain a nearly constant tracer concentration. The lidded chamber was placed inside an incubator with a controlled temperature of $23{ }^{\circ} \mathrm{C}$. We directly mapped the tracer concentration distribution in the 
rock sample after a certain diffusion time, which ranged in hours to one day dependent upon rock properties (e.g., porosity and permeability).

Following the approach of $\mathrm{Hu}$ et al. (2004), unsaturated diffusion tests were conducted for three tuff samples under two initial water saturations (44 and 98\% RH equilibrated). Briefly, a half-element (source-sink) approach was used for diffusion measurement of cm-sized unsaturated tuff cylindrical cores. The source element was vacuum-saturated with a tracer mixture solution (pulling vacuum on dry rock and letting the solution invade evacuated pores afterwards for full saturation), and the sink element was vacuum-saturated with water only (no tracer chemicals). After a preequilibration period inside RH chamber to establish similar water potential between source- and sink-elements to prevent advective transport, the source and sink elements were clamped together to start the diffusion tests. After a certain diffusion duration (in days, dependent upon the level of water saturation; the lower a saturation, the longer the duration), the diffusion test was stopped by separating the source and sink elements. Tracer distributions on the surface, as well as the interior, of both elements were immediately mapped using LA/ICP-MS.

Figure 5 shows representative diffusion profiles for TSw34 samples at two different initial saturations; analysis to obtain the diffusion coefficient $\left(D_{e}\right)$ is underway. Preliminary results show the expected diffusion at high water saturation, while diffusion at lower (initially equilibrated at $44 \% \mathrm{RH}$ ) is significantly hindered. The reduction of diffusion as a function of water saturation is not linear, as discussed in $\mathrm{Hu}$ et al. (2004).
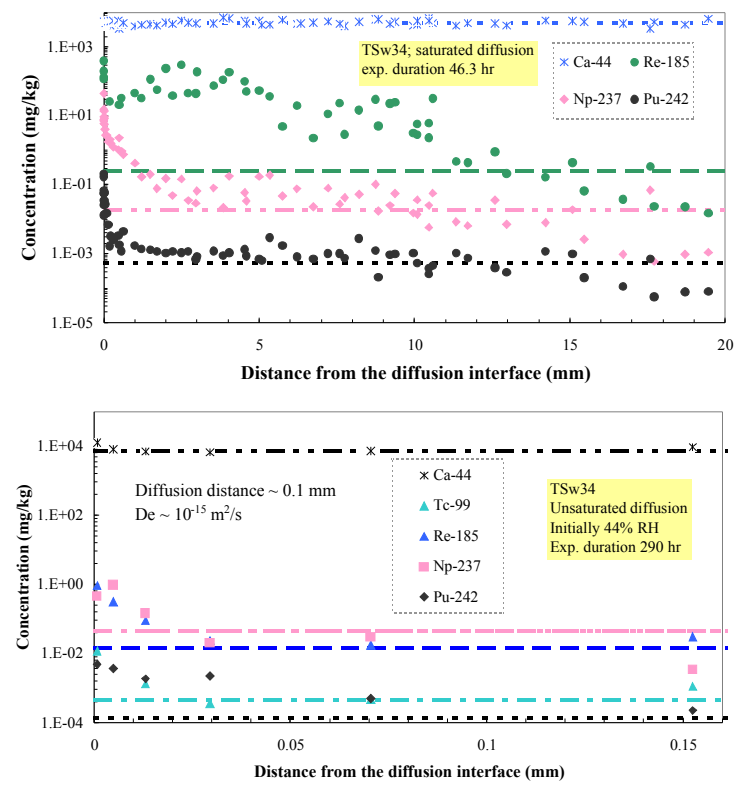

Figure 5. Tracer diffusion into tuff that was initially (A) fully water-saturated, and (B) moist at $44 \% \mathrm{RH}$.

\section{Preferential radionuclide transport in}

altered zones: We performed tracer imbibition into an initially dry TSw34 sample with a 1-mm altered gray zone embedded in highly colored (brown and purple) matrix (Figure 6). Such gray zones were observed to be adjacent to lithophysae and fractures, and identified to be primarily quartz (from alteration of cristobalite) and tridymite (presumably related to vapor phase precipitation).

Using micro-scale profiling of LA/ICP-MS, we surveyed the distribution of radionuclides along (as well as transverse into the unaltered matrix) the altered zone (Figure 6). We found that the altered zone has higher permeability, and less sorption for sorbing radionuclides, than the unaltered matrix, leading to preferential transport along the altered zone. Transverse profiling into the unaltered matrix indicated the limited penetration for strongly sorbing radionuclides, such as $\mathrm{Pu}$ (tabulated values in Figure 7). 


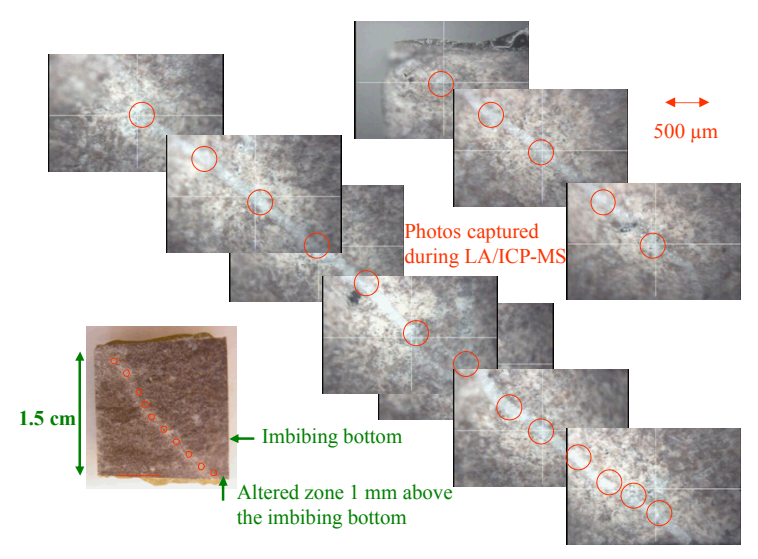

Figure 6. Sampling spots along the altered zone using LA/ICP-MS.

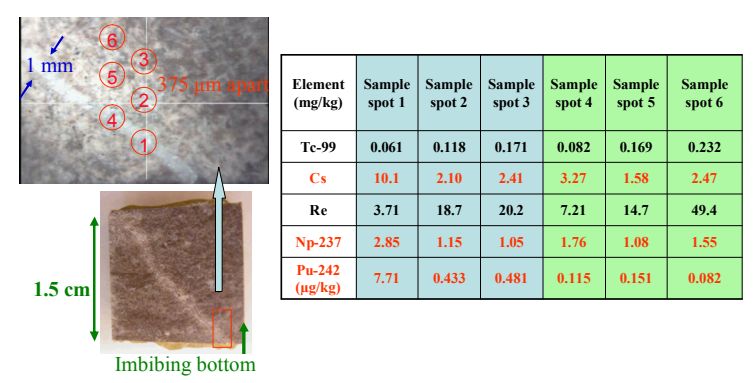

Figure 7. Sampling spots transverse into the unaltered matrix from the altered zone using LA/ICP-MS.

Episodic fracture flow and tracer transport:

We have conducted two fracture flow tests with the tsw34 devitrified rock, one with and one without episodic flow events. We prepared a saw-cut fracture core (length 10.2 $\mathrm{cm}$, diameter $4.4 \mathrm{~cm}$, and fracture aperture $100 \mu \mathrm{m}$ ) for tsw34. The dry core was packed into a flow reactor, flushed with $\mathrm{CO}_{2}$, and then saturated via slow pumping $(0.01$ $\mathrm{mL} / \mathrm{min}$ ) of synthetic groundwater. The fractured core was then flushed with air at $>97 \%$ relative humidity (to simulate in situ unsaturated fractured rock conditions at Yucca Mountain), after which the episodic transport experiment was conducted. Episodic flow involved 4 cycles of tracer solution flow within the fracture, followed by flushing with high humidity air. Each flow episode contained at least one each non-sorbing $\left({ }^{3} \mathrm{H}, \mathrm{Se}, \mathrm{Br}, \mathrm{Mo},{ }^{99} \mathrm{Tc}\right.$, I, and $\mathrm{Re})$ and sorbing ( $\mathrm{Sr}, \mathrm{Cs},{ }^{235} \mathrm{U},{ }^{237} \mathrm{~Np}$, and ${ }^{241} \mathrm{Pu}$ ) tracers (Table 2). Meanwhile, the non-sorbing ${ }^{3} \mathrm{H}$ and $\mathrm{ReO}_{4}{ }^{-}$serve as diffusivity tracers with different aqueous diffusion coefficients.

Table 2. Tracer recipe for episodic transport tests. Highlighted in yellow are important potential dose contributors from nuclear wastes

\begin{tabular}{|c|c|c|c|}
\hline Episode & $\begin{array}{c}\text { Nonsorbing } \\
\text { tracer }\end{array}$ & $\begin{array}{c}\text { Sorbing } \\
\text { tracer }\end{array}$ & $\begin{array}{l}\text { Conc. } \\
\text { (M) }\end{array}$ \\
\hline \multirow{2}{*}{1} & \multirow{2}{*}{$\mathrm{Br}^{-}$} & $\mathrm{Sm} 3+$ & $1.0 \mathrm{E}-04$ \\
\hline & & $\mathrm{Sr}^{2+}$ & $1.0 \mathrm{E}-04$ \\
\hline 2 & $\mathrm{I}^{-}$ & $\mathrm{Cs}^{+}$ & $1.0 \mathrm{E}-04$ \\
\hline \multirow{4}{*}{3} & ${ }^{3} \mathrm{H}$ & & $1.5 \mathrm{E}-10$ \\
\hline & $\mathrm{TcO}_{4}^{-}$ & & $1.0 \mathrm{E}-06$ \\
\hline & \multirow[t]{2}{*}{$\mathrm{ReO}_{4}^{-}$} & & $1.0 \mathrm{E}-05$ \\
\hline & & $\begin{array}{l}\mathrm{U}-235 \\
(\mathrm{VI})\end{array}$ & $1.0 \mathrm{E}-06$ \\
\hline \multirow{4}{*}{4} & $\mathrm{MoO}_{4}{ }^{2-}$ & \multirow[t]{2}{*}{$\mathrm{Ni}^{2+}$} & $1.0 \mathrm{E}-05$ \\
\hline & $\mathrm{SeO}_{4}{ }^{2-}$ & & $1.0 \mathrm{E}-04$ \\
\hline & & $\begin{array}{c}\mathrm{Np}-237 \\
\text { (V) }\end{array}$ & $1.0 \mathrm{E}-05$ \\
\hline & & $\begin{array}{c}\mathrm{Pu}-242 \\
\text { (IV) }\end{array}$ & $5.0 \mathrm{E}-08$ \\
\hline
\end{tabular}

The solution is directly released into the fracture to study the worst-case scenario. Liquid effluent from the flow reactor was collected for multi-elemental analyses using ICP-MS, as well as liquid scintillation counting for ${ }^{3} \mathrm{H}$, to obtain the breakthrough curves of non- or less-retarded tracers. After the flow tests were complete, the flow reactor was opened and the distribution of strongly retarded tracers within the fractured core characterized by LA / ICP-MS. Results 
indicate interacting imbibition, diffusion, and sorption, which will contribute to radionuclide retardation under fracturedominated preferential flow (Figure 8).

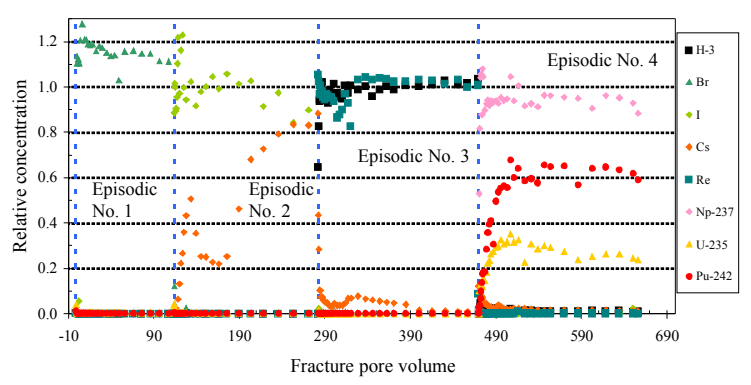

Figure 8. Radionuclide transport in a fractured core column under episodic flow events.

Pore-scale network modeling: Some of the laboratory imbibition tests showed two distinct regimes, having slopes of $1 / 2$ and $1 / 4$ in log-log (cumulative mass imbibed over time) space. "Normal" imbibition gives the slope of $1 / 2$ (the imbibition front moves with the square root of time), but the lower-slope regime appears in rock with poorlyinterconnected porespace. In some cases, imbibition transitioned from low-slope to normal-slope behavior; the opposite was never observed. This behavior was produced in the pore-scale simulations as well, with bonds in the cubic lattice pruned almost to the percolation threshold. It is known from percolation theory that processes operating at scales smaller than some correlation length will have anomalous behavior, while above that scale they will behave normally. The distance from the inlet face at which the slopes cross from anomalous to normal is the correlation distance of percolation theory, and is related to the connectedness of the medium.

Measuring the crossover distance presents two distinct classes of difficulties, which we can classify as measurement and analysis issues. The measurement issues stem from the question, is the crossover length that we measure in the laboratory specific to the sample being tested or the process used? In particular, does it change with the sample size or shape? Theory indicates that squat cores (small length:diameter ratio) will have a smaller crossover length than thin (large length:diameter ratio) ones. Theory further holds that, the closer a sample is to the percolation threshold, the greater will be the variability (both within the sample, and between duplicate samples). As for the process, imbibition and diffusion are sufficiently analogous that they should give equivalent results, but episodic wetting and drying (for example) may show quite different behaviors.

Measurement issues were addressed in several ways. We had already run multiple simulations using cubic lattices of different sizes, ranging from $8^{3}$ to $256^{3}$. This year we expanded our database by running multiple simulations of different shaped lattices, with length:diameter ratios ranging from $1 / 8$ to 8 . These were run at several connectivity values above the percolation threshold. Additionally, in order to cope with the high variance of samples near the percolation threshold, several hundred additional simulations were run at and just above the threshold itself. Simulations are slower the closer they are to the percolation threshold, so significant computer time was involved in these studies.

Measurement issues were compounded by, and their resolution slowed by, the analysis issues. These concern the statistics of the imbibition curves (whether measured or simulated). Specifically, how should the breakpoint (where a curve changes slope) be determined, especially when the data are noisy or the break appears gradual? And, how is the decision best made whether a given curve is "really" one line, or two? 
After trying multiple ad hoc approaches (it had not originally been anticipated that this would be a problem), we reviewed the relevant literature and found that breakpoint regression is an active area of statistics research. One new method - the Weighted Hidden Markov Method - and the classical 3-parameter minimum sum of squares method appear fairly robust with respect to finding the breakpoint. We also compared several test statistics for deciding whether the data represent one line or two. A manuscript detailing this statistical subinvestigation will soon be submitted to a peer-reviewed journal.

Analysis has been delayed by the need to investigate new statistical methods, and by a shortage of funding which necessitated shifting time from other projects. We anticipate resolution of both of these delays in the coming year. Following improved analysis of simulations already run, future simulations can also be chosen.

\section{References:}

Flint, L. E. 2003. Physical and hydraulic properties of volcanic rocks from Yucca Mountain, Nevada. Water Resour. Res., 39(5): Art. No. 1119.

Hu, Q., T.J. Kneafsey, J.S.Y. Wang, L. Tomutsa, and J.J. Roberts. 2004. Characterizing unsaturated diffusion in porous tuff gravels. Vadose Zone Journal, 3(4):1425-1438.

Roberts, S. and B. Viani. 1998.

Determination of mineral abundances in samples from the Exploratory Studies Facility using X-ray diffraction. UCRLID-129748, Lawrence Livermore National Laboratory, Livermore, CA.

\section{Major obstacles to project progress (if any)}

Small budget of this project has been hindering much progress from being made on the research, data reduction and interpretation, and manuscript preparation; other than this, no major obstacles to project progress were identified in the FY06.

\section{Significant changes in research direction (if any)}

No significant changes in research direction were identified in the FY06.

\section{Preliminary results and conclusions}

This research integrates newly developed experimental and modeling methods. Results from water imbibition confirm that the pore space in devitrified rock at Yucca Mountain, where the waste packages may potentially be emplaced, is sparsely connected. Use of standard diffusion models to describe matrix diffusion in the densely fractured devitrified tuff matrices can potentially yield misunderstanding of the diffusion process. Results from the fracture tracer transport test indicate that the interacting processes of imbibition, diffusion, and sorption all contribute to radionuclide retardation under fracturedominated preferential flow.

\section{List of reports and journal articles in preparation and completed}

Ewing, R.P., and Q. Hu. Water imbibition into rock as affected by sample shape, pore connectivity, and antecedent water content. Presented at 2005 ASA-CSSASSSA International Annual Meetings, Salt Lake City, UT, November 6 - 10, 2005.

Hu, Q., Y. Sun, and R.P. Ewing. Radionuclide transport in fractured tuff under episodic flow conditions. Presented at 2005 Fall Meeting for 
American Geophysical Union, San

Francisco, CA, December 5-9, 2005.

Hu, Q., R.P. Ewing, L. Tomutsa, and M.J.

Singleton. 2006. Pore Connectivity,

Episodic Flow, and Unsaturated

Diffusion in Fractured Tuff. Proceedings

of the 11th International High- Level

Radioactive Waste Management

Conference (IHLRWM), pp. 70-76, April 30 - May 4, 2006, Las Vegas, NV.

$\mathrm{Hu}$, Q., R.P. Ewing, and C.I. Steefel.

Interacting processes affecting

contaminant transport in unsaturated

fractured rock. 2006 Annual Meeting for the Geological Society of America, October 22-25, 2006, Philadelphia, PA.

Ewing, R. P. and D. W. Meek. One line or two? A framework for segmented regression. 2006 Annual Meeting of the American Society of Agronomy, Nov. 12-16, Indianapolis, IN.

$\mathrm{Hu}$, Q., T.P. Rose, D.K. Smith, and M.

Zavarin. 2006. Assessing field-scale migration of mobile radionuclides at the Nevada Test Site (in review).

Ewing, R. P. and D. W. Meek. One line or two? A framework for segmented regression (in review).

$\mathrm{Hu}, \mathrm{Q}$., and X. Mao. Applications of laser ablation / inductively coupled plasma-mass spectrometry in studying diffusion, sorption, and transport in unsaturated rock (in preparation).

$\mathrm{Hu}$, Q., and R.P. Ewing. Significance of pore connectivity in chemical transport: Evidence and implications (in preparation).

$\mathrm{Hu}, \mathrm{Q}$. Preferential radionuclide transport in unsaturated fractured tuff (in preparation). 